\title{
High-contrast imaging of massive stars: the example of QZ Car
}

\author{
M. Reggiani, A. Rainot and H. Sana \\ Institute of Astrophysics, KU Leuven, Celestijnlaan 200D, 3001 Leuven, \\ Belgium, (E-mail: maddalena.reggiani@kuleuven.be)
}

Received: October 31, 2019; Accepted: February 3, 2020

\begin{abstract}
The formation of massive stars remains one of the most intriguing questions in astrophysics today. Several formation theories, that could potentially be tested by the multiplicity properties of their outcome, have been proposed. There are, however, observational challenges preventing us from discriminating between the different formation scenarios: massive stars are rare and found at relative large distances from us, they form on short timescales and evolve in multiple stellar systems within the gas-rich environment from which they are born. Taking advantage of the extreme-adaptive optics capabilities of VLT/SPHERE, we observed more than 70 galactic $\mathrm{O}$ stars, about half in the Carina nebula and another half in the galactic field or clusters and associations, aiming at characterizing their multiplicity properties. SPHERE offers unprecedented imaging contrasts which allows us to detect even the faintest companions around massive stars. Here, we illustrate its capabilities by focussing on the high-order multiple system QZ Car.
\end{abstract}

Key words: stars: massive - stars: binaries - stars: formation

\section{Introduction}

How do massive stars form? This is still one of the most important questions in astrophysics today (Tan et al., 2014). From the observational point of view, addressing this question implies facing the fact that massive stars are rare, thus are found at large distances from us, and the timescales of their formation processes are very short. In addition, massive stars are deeply embedded in the cloud of gas and dust they accrete from during their formation. Some of the main formation scenarios that have been proposed are core accretion, competitive accretion, and stellar collisions and merging (Bonnell et al., 1998; Tan et al., 2014). Although most theories agree on the existence of dense and massive accretion disks, which are unstable to fragmentation (Kratter et al., 2010) and may lead to the formation of companions, we lack quantitative model predictions. Finding correlations between multiplicity characteristics may help distinguishing among the different mechanisms of massive star-formation and guide the development of future theoretical models. 
In this context, the binary fraction and multiplicity properties of massive stars have been studied by recent surveys, either through the spectroscopic analysis of young massive stellar clusters (Sana et al., 2012) and OB associations (Kiminki \& Kobulnicky, 2012), or thanks to high-angular resolution observations of massive stars (Sana et al., 2014; Aldoretta et al., 2015). Among those, the VLT SMaSH+ survey (Sana et al., 2014) was the first survey to cover the gap between spectroscopic and classical-imaging observations. Combining optical interferometry (VLTI/PIONIER), aperture masking (NACO/SAM) and imaging (NACO), $\mathrm{SMaSH}+$ searched for bright companions $(\Delta H<4)$ in the $0{ }^{\prime \prime} 001-0{ }^{\prime \prime} 2$ angular separation regime around a large sample of O-type stars, and fainter $(\Delta H<8)$ companions up to $8^{\prime \prime}$. The 260 companions found span the entire range of separations, including several faint stars at angular separations above $1^{\prime \prime}$. After correcting for contamination due to spurious alignment, $\mathrm{SMaSH}+$ measured a multiplicity fraction of $f_{\mathrm{m}}=0.91 \pm 0.03$, demonstrating that almost all massive stars form as part of a multiple stellar system. The high number of faint companions at large separations $\left(>1^{\prime \prime}\right)$, which could correspond to the outer edge of the accretion disk, may result either from fragmented clumps of the accretion disk, or from tidal capture. Probing the existence of such faint companions at closer separations is therefore crucial to understand their origin.

The Extreme AO high-contrast imaging capabilities of the VLT SpectroPolarimetric High-contrast Exoplanet REsearch instrument (SPHERE), combined with dedicated image post-processing algorithms, now allow us to unveil the multiplicity properties of massive stars in a parameter space unexplored so far.

\section{The CHIPS survey}

The Carina High-contrast Imaging Project of massive Stars (CHIPS) aims to probe the multiplicity properties of 92 massive stars in the Carina star-forming region, in the $00^{\prime \prime} 15-5 . " 5$ angular separation range $(\sim 350-12,500 \mathrm{au})$ and down to mass-ratios of 0.03 (on the main sequence). So far, we have carried out SPHERE observations for half of the targets, allowing us to constrain the binary frequency in the separation range probed by SPHERE with a precision better than $7 \%$. Here, we illustrate the capability of SPHERE by using the example of QZ Car.

\subsection{QZ Car}

QZ Car is a high-order multiple system, composed of two spectroscopic binaries (Aa \& Ac), separated by $00^{\prime \prime} 030$, and other three previously imaged companions within 7" Ab, E \& B (Sana et al., 2014; Sanchez-Bermudez et al., 2017).

We observed QZ Car in October 2016 in IRDIFS-EXT mode, which combines the Integral Field Spectrograph and the Infra-Red Dual-beam Imaging and Spectroscopy sub-systems. Such observations enabled us to combine the smaller IFS field-of-view ( f.o.v.), probing the close-environment of the star, with IRDIS 
images that provide information on the local density of faint objects. The observations were conducted in pupil tracking mode, to allow for both Angular (ADI) and Spectral (SDI) Differential Imaging techniques, to disentangle the signal of potential companions from the background noise. After obtaining the reduced (centered, flat fielded, background subtracted) data from the SPHERE data center, we used the Vortex Image Processing package (VIP, Gomez Gonzalez et al., 2017) to apply Principle Component Analysis (PCA). Figure 1 shows the final PCA images for IFS and IRDIS. In total we detected 19 sources with a signal to noise ratio greater than 5 , including the previously known stars $\mathrm{Ab}$ and $\mathrm{E}$, as well as a new companion in the IFS f.o.v., Ad. Although most of the distant and faint sources have high spurious association probabilities (see Rainot et al., submitted), Ab, E, and Ad are all likely bound companions. We extracted the IFS spectrum for Ad and for the central star spectral channel by spectral channel, by adopting a Simplex Nelder-Mead optimization and a Markov-Chain-Monte-Carlo (MCMC). As a YJH flux calibrated spectrum of the central star was not available to flux calibrate the measured spectrum for Ad, we modelled the four components of QZ Car separately using the nonLocal Thermodynamic Equilibrium atmosphere code FASTWIND (Puls et al., 2005). We then compared the flux calibrated spectrum for Ad with model spectral energy distributions from the ATLAS9 LTE atmosphere models (Castelli \& Kurucz, 2004) and the pre-main sequence evolutionary tracks of Siess et al. (2000). We obtain the best agreement for a $2.2 \mathrm{M}_{\odot}$ star with $T_{\text {eff }}=9700 \mathrm{~K}$, $L=25.5 \mathrm{~L}_{\odot}$ and $R=1.77 \mathrm{R}_{\odot}$, corresponding to a spectral type A0. According to Siess et al. (2000) evolutionary tracks, its estimated age is $7.7 \mathrm{Myr}$, in fair agreement with the estimates for QZ Car (Walker et al., 2017).
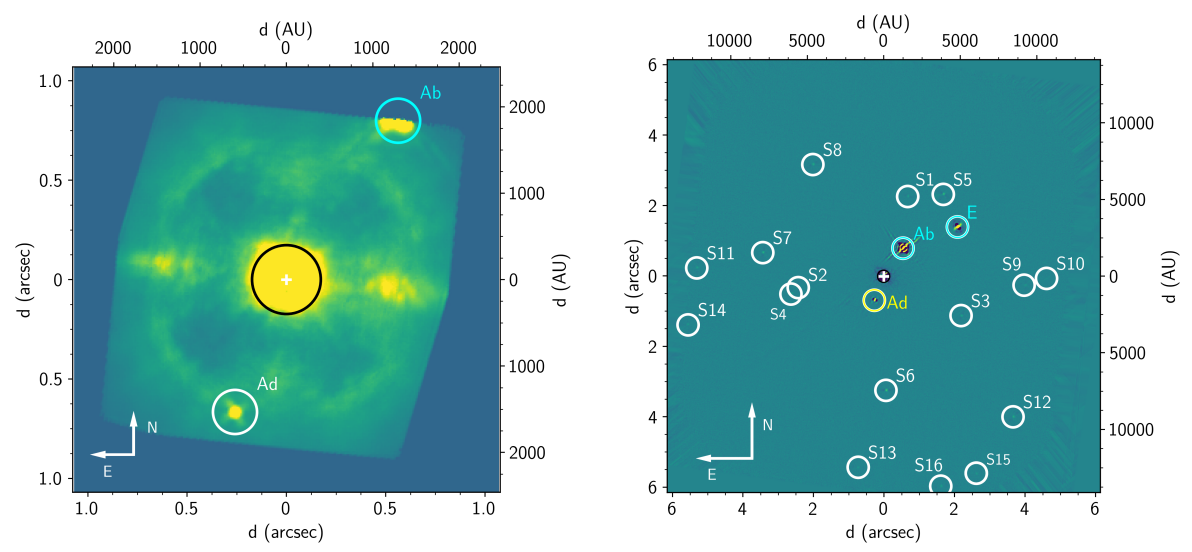

Figure 1. Final PCA images for IFS (left) and IRDIS (right; Rainot et al., submitted). 


\section{Conclusions and future prospects}

We have presented here the first SPHERE observations of QZ Car, a known quadruple system in the Carina region, as part of a survey aiming to characterize the multiplicity properties of $\mathrm{O}$ stars in the Carina region. Combining SPHERE IFS and IRDIS observations, we detected 19 sources in a $12^{\prime \prime} \times 12^{\prime \prime}$ f.o.v., 17 of which are new detections. Although most of the them can be explained by spurious alignment given the stellar density around QZ Car; Ad, Ab, and E are likely physical companions. They are consistent with ages between 4-8 Myr, suggesting that they are coeval with the inner quadruple system. If all three stars are confirmed as bound by proper motion follow-up observations, QZ Car may become one of the highest-order multiple systems known.

Besides 42 stars in the Carina region, we collected SPHERE observations for 15 stars in the the Sco OB association and for a dozen targets from the SMaSH+ catalog. The characterization of all companions in the $0{ }^{\prime \prime} 1-1^{\prime \prime}$ separation regime will soon enable us to compare the multiplicity properties of massive binaries in different environments, as a function of angular separation, cluster age, or environment density.

\section{References}

Aldoretta, E.J. et al. 2015, ApJ 149, 26

Bonnell, I.A., Bate, M.R., Zinnecker, H. 1998, MNRAS 298, 93

Castelli, F., \& Kurucz, R. L. 2004, Modeling of stellar atmosphers, IAU Symp., eds. N. Piskunor et al. [arXiv:astro-ph/0405087]

Gomez Gonzalez, C. A., Wertz, O., Absil, O., et al. 2017, ApJ, 154, 7

Kiminki, D.C., Kobulnicky, H.A. 2012, ApJ 751, 1

Kratter, K.M., Matzner, C.D.; Krumholz, M.R.; Klein, R.I. 2010, ApJ 708, 1585

Puls, J., Urbaneja, M. A., Venero, R., Repolust, T., Springmann, U., Jokuthy, A., Mokiem, M. R. 2005, A\&A 435, 669698

Sana, H., De Mink, S.E., de Koter, A., et al. 2012, Science Vol 337, pp. 444-446

Sana, H., Le Bouquin, J.-B, Lacour, S., Berger, J.-P., et al. 2014, ApJ 215, 1

Sanchez-Bermudez, J., Alberdi, A., Barbá, R., et al. 2017, ApJ 845, 57

Siess, L., Dufour, E., Forestini, M. 2000, A\&A, 358, 593

Tan, J. C., Beltrn, M. T., Caselli, P., et al. 2014, Protostars and Planets VI, Eds. Beuther et al., 149

Walker, W. S. G., Blackford, M., Butland, R., Budding, E. 2017, MNRAS, 470, 2 